\title{
Understanding Mott-Schottky Measurements under Illumination in Organic Bulk Heterojunction Solar Cells
}

\author{
Irene Zonno, ${ }^{1,2}$ Alberto Martinez-Otero, ${ }^{1,2}$ Jan-Christoph Hebig, ${ }^{1}$ and Thomas Kirchartz ${ }^{1,2}$ \\ ${ }^{1}$ IEK5-Photovoltaik, Forschungszentrum Jülich, 52425 Jülich, Germany \\ ${ }^{2}$ Faculty of Engineering and CENIDE, University of Duisburg-Essen, Carl-Benz-Strasse 199, \\ 47057 Duisburg, Germany
}

(Received 16 August 2016; revised manuscript received 31 January 2017; published 24 March 2017)

\begin{abstract}
The Mott-Schottky analysis in the dark is a frequently used method to determine the doping concentration of semiconductors from capacitance-voltage measurements, even for such complex systems as polymer:fullerene blends used for organic solar cells. While the analysis of capacitance-voltage measurements in the dark is relatively well established, the analysis of data taken under illumination is currently not fully understood. Here, we present experiments and simulations to show which physical mechanisms affect the Mott-Schottky analysis under illumination. We show that the mobility of the blend has a major influence on the shape of the capacitance-voltage curve and can be obtained from data taken under reverse bias. In addition, we show that the apparent shift of the built-in voltage observed previously can be explained by a shift of the onset of space-charge-limited collection with illumination intensity.
\end{abstract}

DOI: 10.1103/PhysRevApplied.7.034018

\section{INTRODUCTION}

Impedance-based techniques have been frequently used to study doping concentration [1,2], charge recombination [3-7], and the density of states [8] in various thin-film solar cells including organic solar cells. The most basic impedance-based technique is the capacitance-voltage measurement in the dark, which allows one to derive the doping concentration for sufficiently thick absorber layers $[9,10]$ and gives some information about the amount of band bending in the device. While the capacitance-voltage measurement in the dark is a well-understood method [9-12], capacitance-voltage $(\mathrm{CV})$ measurements under illumination have so far been much more difficult to interpret $[13,14]$ and have shown features that were not straightforward $[15,16]$ to explain with analytical equations. Guerrero et al. [13] and Bisquert et al. [16] observed for P3HT:PCBM-based devices a marked shift in the $C V$ curves with light intensity. In such a case, the use of the Mott-Schottky analysis to calculate the built-in voltage leads to values which are much lower than the value obtained in the dark and may even be negative for high light intensities.

Here, we attempt to explain the features observed in $C V$ measurements under illumination with a combination of the charge-carrier mobility and the photogenerated space charge in the device. Provided that the device is sufficiently thin and not affected by a high doping concentration, we show that the $C V$ curves can be approximately described by a theory of photocapacitance developed by Crandall $[17,18]$ for amorphous silicon solar cells. Through the application of this theory, the mobility can be determined, and $C V$ measurements can be used as complementary method to other techniques for the determination of doping density and mobility $[19,20]$. However, for practical cases, we observe differences between the theory of Crandall and the experimental results that are due to the assumptions required to derive Crandall's equation breaking down in certain cases. For instance, recombination may have a nonnegligible influence on the shape of the current-voltage curve even in short circuit or under reverse bias. We use numerical simulations and analytical estimates of the space-charge-limited photocurrent to show how the capacitance might change relative to Crandall's theory for the example where asymmetric mobilities lead to insufficient charge collection. In particular, we explain why the apparent built-in voltage that is obtained from MottSchottky plots under illumination $[13,16]$ is often substantially lower than the built-in voltage obtained in the dark and sometimes even negative. This initially surprising result can be either attributed to the capacitance originating from photogenerated charge carriers that can build up in low-mobility materials even at reverse bias or from using an inappropriate equivalent circuit for data analysis.

\section{EXPERIMENTAL DETAILS}

The preparation of the polymer solar cells is as follows. We produce devices with the inverted structure glass /ITO/ZnO/ polymers: $\mathrm{PC}_{71} \mathrm{BM} / \mathrm{MoO}_{3} / \mathrm{Ag}$. As polymers, we use PTB7 [poly[(4,8-bis-(2-ethylhexyloxy)-benzo(1,2-b:4,5-b9) dithiophene)-2,6-diyl-alt-(4-(2-ethylhexyl)-3-fluorothieno [3,4-b]thiophene-)-2-carboxylate-2-6-diyl)] ] and PBDTTT-C [poly[(4,8-bis-(2-ethylhexyloxy)-benzo(1,2-b:4,5-b9)dithiophene)-2,6-diyl-alt-(4-(2-ethylhexanoyl)-thieno[3,4-b]thiophene-)-2-6-diyl)]]. The active layer of the PTB7: $\mathrm{PC}_{71} \mathrm{BM}\left([6,6]\right.$-phenyl- $\mathrm{C}_{71}$-butyric acid methyl ester $)$ devices is prepared using a solution of $1: 1.5 \mathrm{wt} \%$ in 
chlorobenzene at a concentration of $25 \mathrm{mg} / \mathrm{mL}$ to which the additive 1,8-diiodooctane ( $3 \%$ by volume) is subsequently added. For the PBDTTT-C-based devices, a 1:1-wt \% ratio of PBDTTT-C to $\mathrm{PC}_{71} \mathrm{BM} 30-\mathrm{mg} / \mathrm{mL}$ chlorobenzene solution is used. The $\mathrm{ZnO}$ layer is spin coated on indium-tinoxide-covered glass substrates sonicated in acetone and isopropanol. The polymer: $\mathrm{PC}_{71} \mathrm{BM}$ solution is deposited on top by spin coating at 1600 or $800 \mathrm{rpm}$ for the PTB7- and PBDTTT-C-based devices, respectively. Finally, $\mathrm{MoO}_{3}$ $(7 \mathrm{~nm})$ and $\mathrm{Ag}(100 \mathrm{~nm})$ are evaporated in high vacuum as cathode.

The characterization of the $\mathrm{PTB} 7: \mathrm{PC}_{71} \mathrm{BM}$ and PBDTTT-C: $\mathrm{PC}_{71} \mathrm{BM}$ devices is carried out under simulated AM1.5 irradiation $\left(100 \mathrm{~mW} / \mathrm{cm}^{2}\right)$ using a xenon lamp as light source. The capacitance measurements are performed with a Gamry Interface 1000 potentiostat at a fixed frequency of $5 \mathrm{kHz}$.

\section{THE MODEL}

At short circuit, the quasi-Fermi-levels of a diode are equal at the two contacts, i.e., the voltage is zero; however, they are not necessarily equal in the bulk of the device, as shown in Fig. 1. Instead, depending on the amount of illumination and the charge-carrier mobilities, charge carriers can build up in the device. Figure 1 illustrates the effect of charge-carrier mobility on the energy band diagram calculated under 1 -sun illumination at $0 \mathrm{~V}$ (shortcircuit conditions).

The data were obtained performing frequency-dependent drift-diffusion simulations with the software SCAPS [21,22] developed by the group of Marc Burgelman at the University of Gent. The parameters used in the simulations can be found in the Supplemental Material [23] Table SI. The higher the illumination and the lower the mobilities,

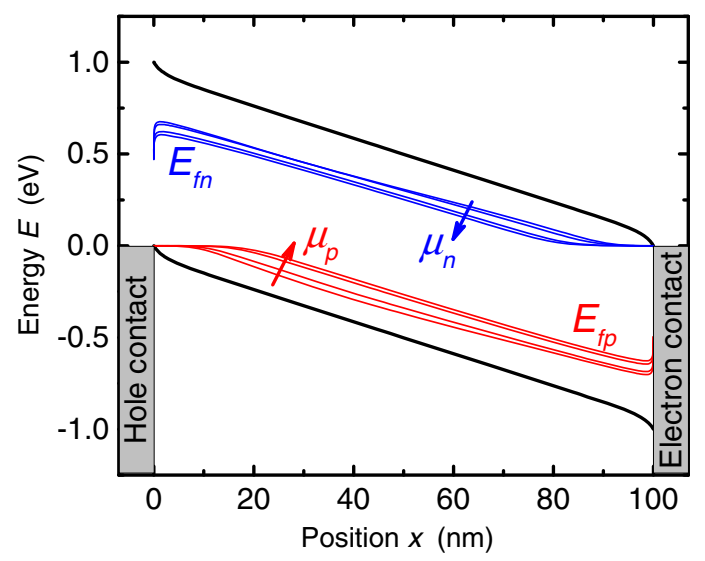

FIG. 1. Band diagram of a 100-nm-thick intrinsic solar cell under 1-sun illumination and short-circuit conditions (applied voltage $V_{\text {ext }}=0 \mathrm{~V}$ ) for different electron and hole mobilities. The quasi-Fermi-level splitting $E_{f n}-E_{f p}$ in the active layer increases progressively with decreasing mobility and the chargecarrier densities change consequently. the more charge carriers will exist at short circuit in a device and will, therefore, contribute to its capacitance. Thus, it is evident that a measurement of either the charge density or the capacitance under illumination will provide information about the charge-carrier mobilities. This concept has been used previously in the time domain, for instance, by Shuttle et al. [24] to determine the mobility in organic solar cells by measuring the extracted charge density under illumination at short circuit. Equivalent information can be obtained using capacitance measurements under illumination and short circuit or reverse bias. Crandall [17] derived an expression for the photocapacitance of a $p-i-n a-\mathrm{Si}: \mathrm{H}$ solar cell which can also apply to organic solar cells under certain circumstances. The derivation in Ref. [17] relies on three assumptions in order to be valid. The assumptions are that recombination has to be negligible, the photocurrent can be approximated by a drift current (diffusion is neglected), and the electric field in the device is constant and equal to $\left(V_{\mathrm{BI}^{-}}-V\right) / d$ where $V_{\mathrm{BI}}$ is the built-in voltage, and $d$ is the active-layer thickness. These assumptions imply that space-charge effects due to, e.g., asymmetric mobilities [25-28], doping [11,29-31], or asymmetric contact barriers [32] are not included in the theory. These effects are more likely to matter in an organic solar cell of a given thickness as opposed to an amorphous $\mathrm{Si}$ solar cell because the relative permittivity of the organic layers is small, and space charge has a stronger influence on the electric field. It is important to note that the breakdown of the assumptions of the negligible recombination rate and constant electric field used in the derivation are important later to explain deviations from Crandall's equation observed in experiment. The expression for the photocapacitance [17]

$$
C_{\mathrm{ph}}=\frac{q G d^{3}}{2\left(\mu_{p}+\mu_{n}\right)\left(V_{\mathrm{BI}}-V\right)^{2}}
$$

shows how its value depends on the light intensity through the generation rate $G$, the sample thickness $d$, the built-in voltage $V_{\mathrm{BI}}$, and the applied dc bias $V$, as well as the electron and hole mobilities $\mu_{n}$ and $\mu_{p}$. Thus, given the thickness of the active layer, the light intensity, and the effective electric field, the value of the capacitance is uniquely determined by the mobilities $\mu_{n}$ and $\mu_{p}$. Equation (1) means that knowing the sample thickness and the generation rate, it is possible to extract the mobility directly from capacitance-voltage measurements under illumination. The total measured capacitance

$$
C_{\mathrm{tot}}=C_{\mathrm{ph}}+C_{\mathrm{dark}}
$$

is the sum of the photocapacitance $C_{\mathrm{ph}}$ and the dark capacitance $C_{\text {dark }}$ which includes the geometrical capacitance. It is possible to rewrite Eq. (1) in order to emphasize the proportionality between the reciprocal of the square root of the photocapacitance and the applied bias $V$. We obtain a straight line if we plot 
$\frac{1}{\sqrt{C_{\mathrm{ph}}}}=\frac{1}{\sqrt{C_{\mathrm{tot}}-C_{\mathrm{dark}}}}=\frac{\sqrt{2\left(\mu_{n}+\mu_{p}\right)}}{\sqrt{q G d^{3}}}\left(V_{\mathrm{BI}}-V\right)$

versus the applied dc bias whose slope can be used to determine the mobility. Here, $\mu$ refers to the average mobility. When the same capacitance-voltage measurement is performed under different light intensities, the plot of $\left(C_{\text {tot }}-C_{\text {dark }}\right)^{-1 / 2}$ versus the applied dc bias can be replaced by the plot of $\left(C_{\mathrm{ph} \text {,norm }}\right)^{-1 / 2}$ versus the applied bias, where the thickness and generation-rate-normalized photocapacitance is defined as

$$
C_{\mathrm{ph}, \text { norm }}=\frac{4 C_{\mathrm{ph}}}{q G d^{3}} .
$$

According to Eq. (3) and assuming $\mu_{p}=\mu_{n}$, this plot should lead to a series of overlapping straight lines whose slope can be used to calculate mobility.

\section{RESULTS AND DISCUSSION}

In order to test the validity of Eq. (1), capacitance-voltage measurements in the dark and as a function of light intensity are performed on solar cells composed of PTB7 blended with $\mathrm{PC}_{71} \mathrm{BM}$ and solar cells composed of PBDTTT-C blended with $\mathrm{PC}_{71} \mathrm{BM}$. All the devices are fabricated using the inverted architecture glass/ITO/ZnO/polymers: $\mathrm{PC}_{71} \mathrm{BM} /$ $\mathrm{MoO}_{3} / \mathrm{Ag}$. The PTB7-based devices are $190 \mathrm{~nm}$ thick, while the PBDTTT-C-based devices are $170 \mathrm{~nm}$ thick.

It is important to stress the fact that when a capacitancevoltage measurement is performed, the quantity recorded as function of voltage is the total impedance of the device at a certain frequency from which the capacitance can later be extracted. In order to analyze measurements done as a function of voltage for a single frequency, the device has to be modeled with a simple equivalent circuit consisting of a resistor and a capacitor. In principle, there are two ways to connect these: in series, which we call the $C_{s} R_{s}$ mode, or in parallel, which we call the $C_{p} R_{p}$ mode. At reverse bias or short circuit, where we mostly analyze our data, the resistance of the diode in parallel to the capacitor $C$ is large and certainly not negligible relative to $C$. Therefore, the $C_{p} R_{p}$ mode is certainly the more sensible one for the analysis of capacitance-voltage measurements. However, the use of the $C_{s} R_{s}$ leads to quite peculiar results, especially under illuminations that are relevant to the analysis of $C V$ measurements. Therefore, in the following, we briefly sketch how these different modes lead to different results for the resulting capacitance.

In the $C_{s} R_{s}$ mode, the total impedance $Z_{s, \text { tot }}$ is simply the sum of the impedance of the resistor $Z_{R}$ and the impedance of the capacitor $Z_{C}\left(Z_{s, \text { tot }}=Z_{R}+Z_{C}\right)$, while in the $C_{p} R_{p}$ mode, the inverse of $Z_{p \text {,tot }}$ is equal to the sum of the inverse of the $Z_{R}$ and the inverse of $Z_{C}\left(1 / Z_{p \text {,tot }}=1 / Z_{R}+1 / Z_{C}\right)$. Thus, in the former case, the capacitance $C_{s}$ can be calculated from the imaginary part of the total impedance $Z_{s, \text { tot }}$ via

$$
\operatorname{Im}\left(Z_{s, \mathrm{tot}}\right)=-\frac{1}{\omega C_{s}},
$$

and in the latter case, the capacitance $C_{p}$ from the imaginary part of the total impedance $Z_{p, \text { tot }}$,

$$
\operatorname{Im}\left(Z_{p, \text { tot }}\right)=-\frac{\omega C_{p} R_{p}^{2}}{1+\omega^{2} C_{p}^{2} R_{p}^{2}},
$$

where $\omega$ is the angular frequency, and $R_{p}$ the value of the resistor connected in parallel with the capacitor $C_{p}$. The two analysis modes normally provide different capacitance values, as shown in Fig. 2, which presents the $C V$
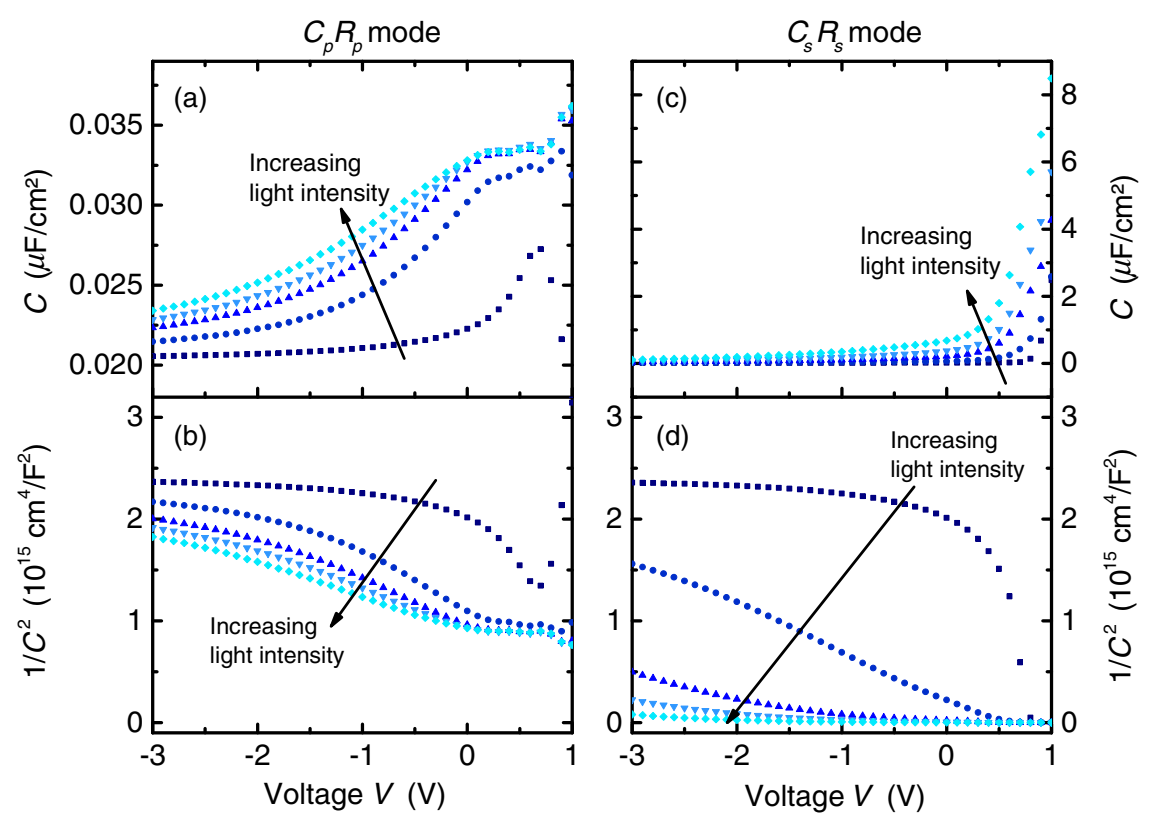

FIG. 2. (a),(c) Capacitance $C$ and (b),(d) $C^{-2}$ versus applied bias for different light intensities (dark, 0.2, 0.5, 0.7, 1 sun) obtained measuring a PBDTTT-C: $\mathrm{PC}_{71} \mathrm{BM}$ (1:1.5 wt \%) device. The panels on the left $[(a),(b)]$ show the case where a capacitor in parallel with a resistor is used to evaluate the data, while the panels on the right $[(\mathrm{c}),(\mathrm{d})]$ show the case where a resistor in series with a capacitor is used to evaluate the data. The data differ drastically, especially with respect to their light-intensity dependence. Particularly, the Mott-Schottky plot in the $C_{s} R_{S}$ mode shows a strong effect of the illumination on the data. 
characteristics and $C^{-2} V$ characteristics (Mott-Schottky plot) obtained by calculating the capacitance from the same impedance measurements on a PBDTTT-C: $\mathrm{PC}_{71} \mathrm{BM}$ $(1: 1.5 \mathrm{wt} \%)$ sample. Note that Figs. 2(a) and 2(c) have a $y$-axis scale which differs by 2 orders of magnitude.

Equating the right sides of Eqs. (5) and (6) and solving for $C_{s}$ leads to

$$
C_{s}=\frac{1}{\omega^{2} C_{p} R_{p}^{2}}+C_{p}
$$

Thus, the difference between the capacitance $C_{s}$ derived using the $C_{s} R_{s}$ mode and the capacitance $C_{p}$ derived using the $C_{p} R_{p}$ mode mostly depends on the value of the resistance $R_{p}$. This value is not constant but depends on the light intensity: the higher the light intensity, the smaller is $R_{p}$. This means that when the light intensity increases, $R_{p}$ changes as well becoming smaller, and, consequently, the difference between $C_{s}$ and $C_{p}$ gets bigger. This tendency is also shown in Fig. 2 where it is evident that the difference between $C_{s}$ and $C_{p}$ becomes larger as the light intensity increases, while it is close to zero in the case of measurements performed in the dark and under reverse bias. Since the $C_{p} R_{p}$ mode is physically more meaningful than the $C_{s} R_{s}$ mode, all the following presented capacitance data are obtained with the $C_{p} R_{p}$ analysis mode.

Figure 3(a) shows the plot of $\left(C_{\mathrm{ph}, \text { norm }}\right)^{-1 / 2}$ versus applied bias for different light intensities $(0.2,0.5,0.7,1$ sun) obtained measuring the PBDTTT-C-based device, whereas Fig. 3(d) shows the same plot for the PTB7-based device.

It can be seen that in both cases the lines corresponding to the different light intensities are not overlapping, as predicted by Eq. (3). However, all the lines are parallel, indicating that the mobility values extracted from the linear fit remain roughly the same as a function of light intensity. The mobility for the PBDTTT-C-based device extracted from the curves in Fig. 3(a) exhibits a nearly constant value equal to $1.24 \times 10^{-5}+/-1.3 \times 10^{-6} \mathrm{~cm}^{2} \mathrm{~V}^{-1} \mathrm{~s}^{-1}$, which is in agreement with literature values for the mobilities measured with other techniques such as charge extraction by linearly increasing voltage [33] and simple charge extraction [34]. The mobility for the PTB7: $\mathrm{PC}_{71} \mathrm{BM}$ sample extracted from the curves in Fig. 3(d) presents a nearly constant value of $1.25 \times 10^{-4}+/-3.9 \times 10^{-5} \mathrm{~cm}^{2} \mathrm{~V}^{-1} \mathrm{~s}^{-1}$, which is in good agreement with the literature values measured with other methods such as open-circuit corrected charge-carrier extraction [35] and time of flight [36]. The possibility to estimate the mobility even in the case of parallel lines is verified by means of numerical simulations performed using the software SCAPS (see the Supplemental Material [23]). However, it is important to note that when the mobilities are asymmetric (and at least one is fairly low), the value extracted using this method is much closer to the value of the lowest mobility than to the average mobility. In order to gain insight into this behavior, the $C V$ characteristics under illumination are analyzed [Figs. 3(b), 3(c), 3(e), and 3(f)]. In Figs. 3(b) and 3(e), the capacitance $C$ is plotted as a function of applied bias for different light intensities. The behavior of the device capacitance is the typical behavior observed in solar cells and features two main regions: for forward bias, the capacitance increases exponentially with voltage due to the injection of electrons and holes from their respective contacts [8,37]; towards reverse bias, the value of $C$ decreases and slowly approaches the value given by the geometric capacitance for infinitely low light intensities and high reverse biases [16]. In the $C V$ characteristics obtained measuring the PBDTTT-Cbased device, we detect a peak, which becomes more evident
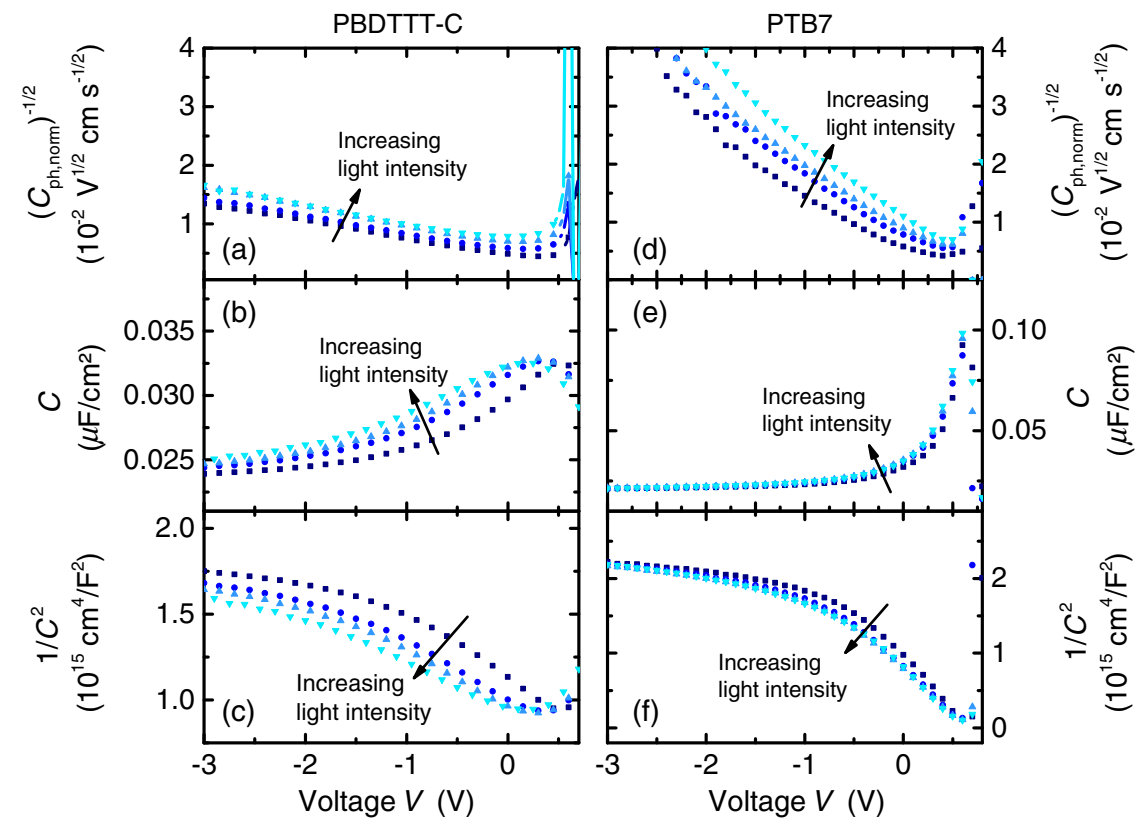

FIG. 3. Capacitance-voltage measurements of (a)-(c) a PBDTTT-C:PC ${ }_{71} \mathrm{BM}$ $(1: 1$ wt \%) solar cell and (d)-(f) a PTB7:PC ${ }_{71} \mathrm{BM}(1: 1.5 \mathrm{wt} \%)$ solar cell as functions of light intensity $(0.2,0.5,0.7,1$ sun). In the case of the PBDTTT-C: $\mathrm{PC}_{71} \mathrm{BM}$ device, the curves of $\left(C_{\mathrm{ph}, \text { norm }}\right)^{-1 / 2}$ versus the applied voltage shift to more positive voltage as the light intensity increases. They remain parallel, however, allowing the extraction of the mobility from their slope. The peak in $C V$ characteristics becomes more left shifted, and the $C^{-2}$ curves shift to more negative voltage as the light intensity increases. In the case of the PTB7: $\mathrm{PC}_{71} \mathrm{BM}$ device, the shift of the peak in $C V$ characteristics and the shift of the $C^{-2}$ curves are less pronounced, and the curves of $\left(C_{\mathrm{ph}, \text { norm }}\right)^{-1 / 2}$ versus the applied voltage slightly move to more positive voltage as the light intensity increases. 
and shifts to lower voltages as the light intensity increases. This systematic shift is equally evident in the plot of $C^{-2}$ versus voltage presented in Fig. 3(c).

The reason for this behavior is found in the asymmetry of the system considered and the consequent nonuniform electric field. Usually, it is possible to make a useful approximation by assuming that the electric field is nearly uniform in the active layer and equal to $\left(V_{\mathrm{BI}}-V\right) / d$. However, there are some cases where the electric field is highly nonuniform, for instance, when the device is highly doped or when the cell thickness increases and the charge transport is strongly unbalanced. Also, traps in the material, asymmetric contact barriers at the anode or cathode, or any combination of the mentioned reasons can lead to nonuniform electric fields which have implications for currentvoltage [11,38] and capacitance-voltage [39] characteristics.

The case with a sufficiently high doping concentration means that the cell shows Mott-Schottky-type behavior both in the dark and under illumination, as shown in the Supplemental Material [23] Fig. S4. Thus, it is possible to derive the doping density and the built-in voltage from the plot of $C^{-2}$ versus voltage [10]. Clearly, the solar cells studied here do not show this kind of behavior; therefore, we choose to have a look at one of the other cases that can lead to nonuniform electric fields. For more detailed simulations, we choose as an example the case of high cell thickness and unbalanced charge transport $\left(\mu_{p} \neq \mu_{n}\right)$. If one considers a semiconductor where the hole drift length is much smaller than the electron drift length and which has a sufficiently high active-layer thickness $(d>200 \mathrm{~nm})$ [9], the holes will accumulate in the device under illumination. As a consequence, the electric field increases in the region near the anode, enhancing the extraction of holes, and decreases in the region near the cathode, diminishing the extraction of electrons [25]. That means that the assumption of constant electric field used in Crandall's derivation breaks down. Since almost the entire voltage $V$ drops over the region of hole accumulation, the energy-band diagram shows a parabolic trend, as it is schematically represented in Fig. 4 illustrating the simulated band diagram of an intrinsic solar cell with asymmetric mobilities $\left(\mu_{p}<\mu_{n}\right)$ under 1-sun illumination at $0 \mathrm{~V}$ (short-circuit conditions).

The accumulated holes in the region near the anode cause the formation of positive space charge which limits the photocurrent generated by the device, i.e., the initial assumption that recombination at short circuit or reverse bias is negligible breaks down. In this space-charge-limited regime, the length $L$ of the current-limiting layer and the maximum electrostatically allowed photocurrent $J_{\mathrm{ph}}$ that can be extracted from the device are, respectively, [25]

$$
L=\left(\frac{9 \varepsilon_{0} \varepsilon_{r} \min \left(\mu_{p}, \mu_{n}\right)}{8 q G}\right)^{1 / 4} V^{1 / 2}
$$

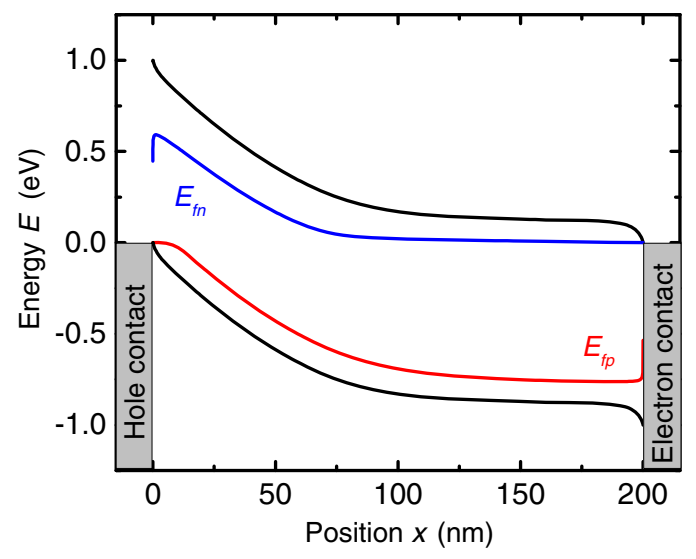

FIG. 4. Band diagram under 1-sun illumination and shortcircuit conditions (applied voltage $V_{\text {ext }}=0 \mathrm{~V}$ ) of an intrinsic solar cell with 200-nm-thick active layer and asymmetric mobilities $\left(\mu_{p}=5 \times 10^{-5} \mathrm{~cm}^{2} \mathrm{~V}^{-1} \mathrm{~s}^{-1}, \mu_{n}=5 \times 10^{-4} \mathrm{~cm}^{2} \mathrm{~V}^{-1} \mathrm{~s}^{-1}\right)$. The band's bending suggests the presence of a nonuniform electric field inside the active layer. In fact, the holes accumulate to a greater extent in the device than in the electrons, which makes the electric field increase in the region near the anode and decrease in the region near the cathode.

$$
J_{\mathrm{ph}}=q\left(\frac{9 \varepsilon_{0} \varepsilon_{r} \min \left(\mu_{p}, \mu_{n}\right)}{8 q}\right)^{1 / 4} G^{3 / 4} V^{1 / 2} .
$$

By assuming that the capacitance scales with the reciprocal of the length $L$ of the current-limiting layer, it follows that in the space-charge-limited regime, the photocapacitance of the solar cell scales with $G^{1 / 4}$. Moreover, according to Eq. (8), the photocapacitance also varies inversely with the square root of the voltage, as in a Schottky barrier. On the other hand, when the length $L$ of the current-limiting layer becomes equal to the device thickness $d$, the photocurrent saturates to the value of $J_{\mathrm{ph}}=q G d$. Under this condition, the electric field can be again considered uniform in the whole active layer, and the expression derived for the photocapacitance is equal to the one presented in Eq. (1). Only in the saturation region, the photocurrent is at its maximum value; i.e., recombination can be neglected, and the photocapacitance follows Crandall's equation. Thus, when the photocurrent $J_{\mathrm{ph}}$ switches from the space-charge-limited regime to the saturation regime, the photocapacitance dependence on the voltage changes consequently. Taking into account that the transition between the two photocurrent regimes takes place when the space-charge-limited photocurrent is equal to the saturation photocurrent, the voltage $V_{p}$ at which $J_{\mathrm{ph}}$ changes can be determined via

$$
V_{p}=\frac{G^{1 / 2} d^{2}}{\left(\frac{9 \varepsilon_{0} \varepsilon_{r} \min \left(\mu_{p}, \mu_{n}\right)}{8 q}\right)^{1 / 2}} .
$$



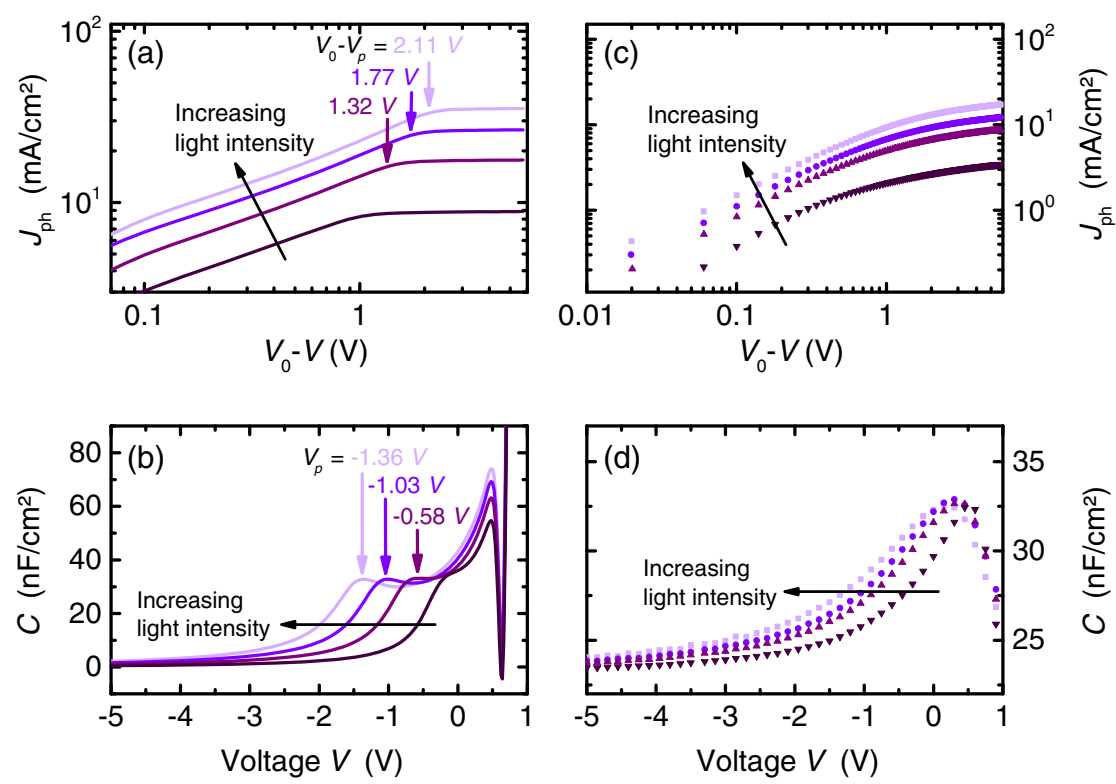

FIG. 5. Graphs showing the $J_{\mathrm{ph}}-\left(V_{0}-V\right)$ characteristics and $C V$ characteristics obtained (a) and (b) from the simulations of an intrinsic solar cell with 200-nm-thick active layer and unbalanced mobilities $\left(\mu_{p}=5 \times 10^{-5} \mathrm{~cm}^{2} \mathrm{~V}^{-1} \mathrm{~s}^{-1}, \quad \mu_{n}=5 \times\right.$ $10^{-4} \mathrm{~cm}^{2} \mathrm{~V}^{-1} \mathrm{~s}^{-1}$ ) and (c),(d) from measuring a PBDTTT-C: $\mathrm{PC}_{71} \mathrm{BM}(1: 1 \mathrm{wt} \%)$ device. (a),(c) Incident light-intensity dependence of the photocurrent $J_{\mathrm{ph}}$ versus the effective applied voltage $\left(V_{0}-V\right)$. The change in the slope underlines the transition from the space-charge-limited regime to the saturation regime. As the arrows indicate, the voltage $V_{p}$ corresponding to this transition decreases as the light intensity increases. (b),(d) Capacitance versus voltage as a function of light intensity. The peak shifts towards more negative voltage as the light intensity increases, and, therefore, the voltage $V_{p}$ decreases consequently like the arrows point out.
Since from Eq. (7) it becomes clear that the voltage at which $J_{\text {ph }}$ changes depends on the light intensity, it is reasonable to expect that the position of the peak in the $\mathrm{CV}$ characteristic varies as well moving towards more negative voltages as the light intensity increases. This behavior can be clearly observed in Figs. 5(a) and 5(b) which show, respectively, the simulated $J_{\mathrm{ph}}-\left(V_{0_{-}} V\right)$ and $C V$ characteristics of an intrinsic solar cell with unbalanced mobilities $\left(\mu_{p}<\mu_{n}\right)$. Here, $V_{0}$ refers to the compensation voltage, i.e., the voltage at which $J_{\mathrm{ph}}=0$. In these figures, the transition between the two regions is underlined by a change in the slope in the simulated $J_{\mathrm{ph}}-\left(V_{0^{-}}-V\right)$ characteristics and by a peak in the simulated $C V$ characteristics. As the light intensity increases, the voltage $V_{p}$ at which the transition occurs decreases. The same trend, even if less marked, can be detected in Figs. 5(c) and 5(d) showing, respectively, the $J_{\mathrm{ph}^{-}}\left(V_{0}-V\right)$ and $C V$ characteristics of a PBDTTT-C: $\mathrm{PC}_{71} \mathrm{BM}(1: 1 \mathrm{wt} \%)$ device, i.e., with a nonideal donor-acceptor ratio [34]. However, in this case, the active layer is thinner than the one set for the simulations; therefore, the current-limiting region $L$ becomes equal to the device thickness $d$ already at forward bias, and the photocurrent almost immediately saturates to its maximum value.

Note, however, that it is not possible to assign the trends in experimental data where $C V$ curves shift with illumination to, e.g., asymmetric mobilities. The shifts in the $C V$ curves with light intensity are indeed due to violations of the assumptions needed to derive Crandall's model and asymmetric, and low mobilities are only one of several explanations that one cannot easily distinguish without substantial further efforts. The purpose of Fig. 5 is, therefore, to show that asymmetric mobilities are one possible explanation and not to suggest that they are the (most likely) explanation for the data in Fig. 3 or any other data where the $C V$ curves shift with illumination.

\section{CONCLUSION}

In this paper, we propose an explanation for the features observed in capacitance-voltage measurements under illumination of organic solar cells. We show that when the device is sufficiently thin and not affected by a high doping concentration, the $C V$ curves can be approximately described by a theory of photocapacitance developed by Crandall for amorphous silicon solar cells. We explain the differences between the theory of Crandall and the experimental results using numerical simulations and analytical estimates of the space-charge-limited photocurrent. Finally, we point out that the capacitance originating from photogenerated charge carriers makes the apparent built-in voltage obtained from Mott-Schottky plots under illumination lower than the built-in voltage obtained in the dark.

\section{ACKNOWLEDGMENTS}

The authors like to thank Matt White (University of Vermont, Burlington) and Jao van de Lagemaat (NREL, Golden, Colorado) for discussions and sharing of unpublished data that inspired part of the present work. We like to thank Eduardo Coello-Siqueiros for preparation of the PBDTTT-C:PCBM sample. J. C. H. and T. K. acknowledge support from the Bavarian Ministry for Economics, Media, Energy and Technology via the project "Recombination in lead-free perovskite solar cells". T. K. acknowledges support via the Helmholtz Energy Alliance Hybrid PV and from the Deutsche Forschungsgemeinschaft (Grant No. KI1571/2-1). 
[1] I. Mora-Seró, G. Garcia-Belmonte, P. P. Boix, M. A. Vázquez, and J. Bisquert, Impedance spectroscopy characterisation of highly efficient silicon solar cells under different light illumination intensities, Energy Environ. Sci. 2, 678 (2009).

[2] J. V. Li, A. M. Nardes, Z. Liang, S. E. Shaheen, B. A. Gregg, and D. H. Levi, Simultaneous measurement of carrier density and mobility of organic semiconductors using capacitance techniques, Org. Electron. 12, 1879 (2011).

[3] F. Fabregat-Santiago, G. Garcia-Belmonte, I. Mora-Seró, and J. Bisquert, Characterization of nanostructured hybrid and organic solar cells by impedance spectroscopy, Phys. Chem. Chem. Phys. 13, 9083 (2011).

[4] L. Xu, Y.-J. Lee, and J. W. P. Hsu, Charge collection in bulk heterojunction organic photovoltaic devices: An impedance spectroscopy study, Appl. Phys. Lett. 105, 123904 (2014).

[5] J. I. Basham, T. N. Jackson, and D. J. Gundlach, Predicting the $J-V$ curve in organic photovoltaics using impedance spectroscopy, Adv. Energy Mater. 4, 1400499 (2014).

[6] L. C. C. Elliott, J. I. Basham, K. P. Pernstich, P. R. Shrestha, L. J. Richter, D. M. DeLongchamp, and D. J. Gundlach, Probing charge recombination dynamics in organic photovoltaic devices under open-circuit conditions, Adv. Energy Mater. 4, 1400356 (2014).

[7] I. Mora-Seró, Y. Luo, G. Garcia-Belmonte, J. Bisquert, D. Muñoz, C. Voz, J. Puigdollers, and R. Alcubilla, Recombination rates in heterojunction silicon solar cells analyzed by impedance spectroscopy at forward bias and under illumination, Sol. Energy Mater. Sol. Cells 92, 505 (2008).

[8] G. Garcia-Belmonte, P. P. Boix, J. Bisquert, M. Sessolo, and H. J. Bolink, Simultaneous determination of carrier lifetime and electron density-of-states in P3HT:PCBM organic solar cells under illumination by impedance spectroscopy, Sol. Energy Mater. Sol. Cells 94, 366 (2010).

[9] T. Kirchartz, W. Gong, S. A. Hawks, T. Agostinelli, R. C. I. MacKenzie, Y. Yang, and J. Nelson, Sensitivity of the MottSchottky analysis in organic solar cells, J. Phys. Chem. C 116, 7672 (2012).

[10] J. Heath and P. Zabierowski, in Advanced Characterization Techniques for Thin Film Solar Cells, edited by D. AbouRas, T. Kirchartz, and U. Rau (Wiley-VCH, Weinheim, 2011).

[11] F. Deledalle, T. Kirchartz, M. S. Vezie, M. Campoy-Quiles, P. S. Tuladhar, J. Nelson, and J. R. Durrant, Understanding the Effect of Unintentional Doping on Transport Optimization and Analysis in Efficient Organic Bulk-Heterojunction Solar Cells, Phys. Rev. X 5, 011032 (2015).

[12] S. Khelifi, K. Decock, J. Lauwaert, H. Vrielinck, D. Spoltore, F. Piersimoni, J. Manca, A. Belghachi, and M. Burgelman, Investigation of defects by admittance spectroscopy measurements in poly (3-hexylthiophene):(6,6)phenyl C61-butyric acid methyl ester organic solar cells degraded under air exposure, J. Appl. Phys. 110, 094509 (2011).

[13] A. Guerrero, S. Chambon, L. Hirsch, and G. GarciaBelmonte, Light-modulated TiOx interlayer dipole and contact activation in organic solar cell cathodes, Adv. Funct. Mater. 24, 6234 (2014).

[14] Y. Ning, L. Lv, Y. Lu, C. Zhang, Y. Fang, A. Tang, Y. Hu, Z. Lou, F. Teng, and Y. Hou, Effects of photo-induced defects on the performance of PBDTTT-C/PC 70 BM solar cells, Phys. Status Solidi 9, 120 (2015).

[15] B. Qi, Z.-G. Zhang, and J. Wang, Uncovering the role of cathode buffer layer in organic solar cells, Sci. Rep. 5, 7803 (2015).

[16] J. Bisquert, G. Garcia-Belmonte, A. Munar, M. Sessolo, A. Soriano, and H. J. Bolink, Band unpinning and photovoltaic model for P3HT:PCBM organic bulk heterojunctions under illumination, Chem. Phys. Lett. 465, 57 (2008).

[17] R. S. Crandall, Modeling of thin film solar cells: Uniform field approximation, J. Appl. Phys. 54, 7176 (1983).

[18] R. S. Crandall, Photocapacitance of mobile carriers in hydrogenated amorphous silicon solar cells, Appl. Phys. Lett. 42, 451 (1983).

[19] O. J. Sandberg, M. Nyman, and R. Österbacka, Direct determination of doping concentration and built-in voltage from extraction current transients, Org. Electron. 15, 3413 (2014).

[20] M. Nyman, S. Dahlström, O. J. Sandberg, and R. Österbacka, Unintentional bulk doping of polymer-fullerene blends from a thin interfacial layer of $\mathrm{MoO}_{3}, \mathrm{Adv}$. Energy Mater. 6, 1600670 (2016).

[21] M. Burgelman, P. Nollet, and S. Degrave, Modelling polycrystalline semiconductor solar cells, Thin Solid Films 361-362, 527 (2000).

[22] M. Burgelman, K. Decock, S. Khelifi, and A. Abass, Advanced electrical simulation of thin film solar cells, Thin Solid Films 535, 296 (2013).

[23] See Supplemental Material at http://link.aps.org/ supplemental/10.1103/PhysRevApplied.7.034018 for the parameters used in the simulations and additional data from simulations and experiments.

[24] C. G. Shuttle, R. Hamilton, J. Nelson, B. C. O’Regan, and J. R. Durrant, Measurement of charge-density dependence of carrier mobility in an organic semiconductor blend, Adv. Funct. Mater. 20, 698 (2010).

[25] V. D. Mihailetchi, J. Wildeman, and P. W. M. Blom, SpaceCharge Limited Photocurrent, Phys. Rev. Lett. 94, 126602 (2005).

[26] A. Armin, A. Yazmaciyan, M. Hambsch, J. Li, P. L. Burn, and P. Meredith, Electro-optics of conventional and inverted thick junction organic solar cells, ACS Photonics 2, 1745 (2015).

[27] T. Kirchartz, T. Agostinelli, M. Campoy-Quiles, W. Gong, and J. Nelson, Understanding the thickness-dependent performance of organic bulk heterojunction solar cells: The influence of mobility, lifetime, and space charge, J. Phys. Chem. Lett. 3, 3470 (2012).

[28] J. G. Tait, U. W. Paetzold, D. Cheyns, M. Turbiez, P. Heremans, and B. P. Rand, Interfacial depletion regions: Beyond the space charge limit in thick bulk heterojunctions, ACS Appl. Mater. Interfaces 8, 2211 (2016).

[29] J. Wang, L. Xu, Y.-J. Lee, M. De Anda Villa, A. V. Malko, and J. W. P. Hsu, Effects of contact-induced doping on the behaviors of organic photovoltaic devices, Nano Lett. 15, 7627 (2015).

[30] J. C. Blakesley, F. A. Castro, W. Kylberg, G. F. A. Dibb, C. Arantes, R. Valaski, M. Cremona, J. S. Kim, and J. S. Kim, Towards reliable charge-mobility benchmark measurements for organic semiconductors, Org. Electron. 15, 1263 (2014). 
[31] V. A. Trukhanov, V. V. Bruevich, and D. Y. Paraschuk, Effect of doping on performance of organic solar cells, Phys. Rev. B 84, 205318 (2011).

[32] O. J. Sandberg, M. Nyman, and R. Österbacka, Effect of Contacts in Organic Bulk Heterojunction Solar Cells, Phys. Rev. Applied 1, 024003 (2014).

[33] Y. (Michael) Yang, W. Chen, L. Dou, W.-H. Chang, H.-S. Duan, B. Bob, G. Li, and Y. Yang, High-performance multiple-donor bulk heterojunction solar cells, Nat. Photonics 9, 190 (2015).

[34] S. A. Hawks, F. Deledalle, J. Yao, D. G. Rebois, G. Li, J. Nelson, Y. Yang, T. Kirchartz, and J. R. Durrant, Relating recombination, density of states, and device performance in an efficient polymer:fullerene organic solar cell blend, Adv. Energy Mater. 3, 1201 (2013).

[35] A. Zusan, B. Gieseking, M. Zerson, V. Dyakonov, R. Magerle, and C. Deibel, The effect of diiodooctane on the charge carrier generation in organic solar cells based on the copolymer PBDTTT-C, Sci. Rep. 5, 8286 (2015).

[36] B. Ebenhoch, S. A. J. Thomson, K. Genevičius, G. Juška, and I. D. W. Samuel, Charge carrier mobility of the organic photovoltaic materials PTB7 and PC71BM and its influence on device performance, Org. Electron. 22, 62 (2015).

[37] G. Garcia-Belmonte, A. Munar, E. M. Barea, J. Bisquert, I. Ugarte, and R. Pacios, Charge carrier mobility and lifetime of organic bulk heterojunctions analyzed by impedance spectroscopy, Org. Electron. 9, 847 (2008).

[38] G. F. A. Dibb, M.-A. Muth, T. Kirchartz, S. Engmann, H. Hoppe, G. Gobsch, M. Thelakkat, N. Blouin, S. Tierney, M. Carrasco-Orozco, J. R. Durrant, and J. Nelson, Influence of doping on charge carrier collection in normal and inverted geometry polymer:fullerene solar cells, Sci. Rep. 3, 3335 (2013).

[39] R. S. Crandall, Modeling of thin-film solar cells: Nonuniform field, J. Appl. Phys. 55, 4418 (1984). 